\title{
Printing Process Parameters Identification System
}

\author{
Zeljko ZELJKOVIC*, Nemanja KASIKOVIC, Stefan DJURDJEVIC, Dragoljub NOVAKOVIC
}

\begin{abstract}
The paper presents the research aimed at setting up and developing a software system for the printing process parameters identification based on modern computer and software systems, algorithmic principles, principles of expert systems construction and advanced learning. Thus, the possibilities of application of contemporary software tools were investigated, which facilitates the process and forms the program structure of the model that uses programming languages based on the expert systems construction principles and tools for the development of system model based on the principles of modern learning. For complex model development, concepts of process knowledge bases with influential process parameters of printing technique have been developed through modelling and construction based on the logic of expert systems with the presentation, use and involvement of experts knowledge in decision making with the evaluation of the impact of individual parameters. In addition to this approach, a module was developed using modern software tools based on an algorithmic principle and a module for identifying printing process parameters using modern platforms based on advanced learning. Sophisticated software model has been made through the research and developed with databases of process parameter identification systems based on modern software tools. This tool enables a significant expedition of the solution resolving, thus improving the graphical production process and the processes of acquiring and expanding knowledge. The model is based on integrative modules: a printing process parameters identification system based on algorithmic program structure systems, a printing process parameters identification system based on expert system building principles, and a printing process parameter identification system based on modern learning systems.
\end{abstract}

Keywords: expert systems; modern learning; press; process parameters; software systems

\section{INTRODUCTION}

The most crucial postulate of offset printing is related to the amount and application of ink to the substrate, which is the basis of colour perception. The visual appearance of the print is the dominant parameter of the observers rating. Therefore many influential parameters on the print as a requirement have the assurance of the high-quality reproduction, that is, obtaining colours in the most natural visual form. Printing machines attain a high technological level, so good process knowledge should be obtained for their operation. The required process knowledge is broad, so it is necessary to find solutions as the new knowledge may be acquired quickly.

The operator of a modern offset printing machine must have specific expertise and practical experience to be able to make appropriate decisions at certain moments that will promptly change the printing process parameters that will improve the quality of reproduction. Often, the operator needs some information to make his decision. This data is related to the process parameters corrections that are derived from the current print. Instructions information may assist the operator and all participants in the stages of graphic production. This kind of data requires specific knowledge which can be presented through knowledge bases developed by different software tools. In such systems, the logic of learning and decision-making is presented in a particular way with the help of computer and software systems which, as a whole, is a specific software solution. After realizing this, the capabilities of identifying the printing process parameters should be developed. The process parameters are related to the production stages of the process, which include prepress, press and postpress. Research in this area is focused explicitly on the process of identifying parameters related to each stage of graphic production. Thus, in the field of prepress, many universal software tools should be used that often need to be adapted for the specific requirements of the next stages of the process. Quality solutions include the integration of process parameter identification for all stages. This is most often done using a database that integrates information management at all stages of the production process. In the press and postpress stages, there is a tendency to obtain useful information about the output parameters from the process stages that serve as a control mechanism. The graphics machine has a large number of process parameters that influence the printing process. A graphic machine is made up of a set of elements and assemblies of system units in order to obtain prints and finished graphic products.

Newly developed software includes the processing of specific data obtained by research [1-7] that allow the formation of base of particulardata relevant to the process of obtaining a graphic product. Existing software is the production and commercially developed and does not go into the depth of research that is relevant to more profoundresearch that can be used in research centres.

\section{RELATED WORK}

Printing processes are only part of the manufacturing flow of graphic products. Nowadays, in the era of high automation of graphic processes, a special place belongs to information technologies from both hardware and software aspects. According to Kiphan [8], the production flow of graphic production is divided into three major production phases, namely prepress, press and postpress. In the realisation phase, there is a considerable number of process parameters that are related to each printing technique, and thus the printing machine. Many systems developed worldwide have been analysed to advance a printing process parameters identification system. Development approaches, tools used, and characteristics of the solutions implemented are discussed. Most of the analysed systems are based on artificial intelligence techniques with expert systems, neural networks and phase logic. Specific diagnostic approaches can be extracted from the analysis.

In Almutawa \& Moon research [9], a CONES neural network expert system is presented, which indicates the visible variables in the setting performed by the machine operator on the principle of neural networks. The system 
allows modelling the knowledge of an experienced operator on a printing machine that reduces the deviation in the colour application, that is, the quality of the print. Based on such experience and knowledge, a decisionmaking base is formed in determining the use of one or more printing inks that are changing, in order to maintain print quality.

In Bergman research [10], control points in the offset printing process are identified, and methods are presented to evaluate the quality of the printing process, namely methods for determining whether the printing form bears the appropriate image and methods for determining the amount of ink applied to the substrate. It is noted that the quality of colour images reproduction in offset printing depends on many parameters. In the end, it is the amount and application of ink that is applied to the substrate.

Tchan, Thompson, \& Manning [11], developed a system that can simulate human estimates of print quality for simple printing, consisting of an image analysis system and trained neural networks. The image analysis system is used to collect and prepare raw data from prints. The neural network is then used to create computer models. The image analysis system and neural network models are subsequently used to predict observers' ratings for a further set of prints that lack supervised training.

Englund \& Verikas 12] have developed an approach to use a camera to measure a colour raster image, together with the parameters of the printing process for the construction of a control system for controlling the colour flow in the offset printing process. Multiple controller models for colour flow are presented. Both direct and reverse process models were used to find the most suitable control signal.

The impact of paper and printing machine parameters on print quality is particularly pronounced. A database containing print and paper parameters has been developed for the needs of the operator [13]. The selection of attributes and parameters is accessed through a developed software tool. The proposed system makes it possible to evaluate print quality through characteristics in the printing process and to relate quality to production parameters.

Various software can be used to interpret process parameter identification, that is, to resolve problems. The suitability of such interpretation belongs to artificial intelligence, expert systems, neural networks, phase logic, genetic algorithms, hybrid systems, distance learning, multimedia, general-purpose software systems and other application systems.

Each of these approaches has its developmental logic with the ability to link knowledge bases in one general application model. There are certain areas in the field of artificial intelligence [14] that develop as separate segments in the form of expert systems, neural networks, phase logic, genetic algorithms and genetic programming and the like hybrid systems. Expert systems make one part of development in artificial intelligence [15] that is based on the logic of thinking of an expert in a particular field where decisions are realised in the form of programming systems with special programming tools. The solutions are mostly dependent on the engineers and experts skill while solving them. The graphic processes are complex processes, and their solutions take place in different technological flows, so it is most appropriate to note the development of solutions based on the logic of expert systems. Problems without algorithmic solutions are mainly implied in solving graphical processes, and it can be said that the flow may be reduced to software solutions based on artificial intelligence. The functioning of human thinking is a constant research challenge without an exact answer as a result of meaningful investigation. Prolog and Lisp are among the most crucial programming languages essential for artificial intelligence. Outstanding programming languages are problem-oriented, Fortran, Pascal, C, etc., object-oriented, C++, Java, etc. or languages for working with symbols, Lisp, Prologue, etc. They enable development but are unsuitable for presenting knowledge or accessing the knowledge base. The Prolog language is most commonly used for the development of expert systems.

Artificial intelligence techniques in the graphic processes are modestly pronounced in the application, but there are many approaches in the use of artificial intelligence in problem-solving.

Neural networks can help the machine operator make appropriate and timely adjustments to the machine to compensate for the colour deviation from the reference values [16] as well as fuzzy logic and genetic algorithms [17].

Based on the integration of AI technology with information communications, manufacturing, and related product technology $\mathrm{Li}, \mathrm{Hou}, \mathrm{Yu}$ et al. proposed new models, means, and forms of intelligent manufacturing, intelligent manufacturing system architecture, and intelligent manufacturing technology system [18].

Varepo, Trapeznikova, Panichkin, Alexander, Roev \& Kulikov developed software for quantitative estimation of coefficients of ink transfer on the printed substrate in offset printing. This software allows calculating the transfer of ink on the substrate between the contacting cylindrical surfaces of the sheet-fed offset printing apparatus with the boundaries deformation [19].

Orlova, Yu, Varepo \& Hodes used open-source software applications for diagnostics of paper - dampening solution printing system parameters in offset printing. This research shows the need for predicting paper parameters interaction with the dampening solution in an offset printing [20].

Villalba-Diez, Schmidt, Gevers, Ordieres-Meré, Buchwitz \& Wellbrock [21] developed a deep neural network to classify optical defects, to ensure a high application potential and to ensure the high-quality control of production-based industries in the increasingly complex environment.

Based on machine vision Zhang, Chen, Gao, Duan \& Jing developed automatic defect detection for web offset printing [22].

He \& Bai [23] reviewed the use of digital twins for product maintenance and fault diagnosis through predictive models such as artificial intelligence.

Chen, He, Gao \& Zhang studied automatic methods for searching feature regions in printed image registration, and the existing problems of the manual marker feature regions [24].

A printing defect classification method based on deep learning and deep models has been presented in Zhang, Li, Li \& Chen research [25]. 
Alom, Taha, Yakopcic, Westberg, Sidike, Nasrin, Hasan, Van Essen, Awwal \& Asari experimental results in research [26] show state-of-the-art performance using deep learning. They compared it to traditional machine learning approaches in the fields of image processing, computer vision, speech recognition, machine translation, art, medical imaging, medical information processing, robotics and control, bioinformatics, natural language processing, cyber security, and many others.

\section{METHODOLOGY}

In the research realisation, modern approaches of solving through various software applications based on advanced software tools such as MS Visual Studio 2010 Professional were used in one segment, and in the other segment the logic of development of expert systems with specific software tools. The concept was developed based on the expert systems development with the input and output data of the expert system and the overall complex model of the system for identifying the printing process parameters. The concept of identification of printing process parameters on the basis of distance learning has been developed as a particularly significant concept applied in scientific research. There are numerous benefits for users as it provides access across a range of devices where mobile devices excel. They can be used both in the processes of the disjointed structure of production plant machines and from dislocated plant sites and other facilities. For the stated concept of software application setup, data were collected, which made knowledge bases on the most critical process parameters. These parameters enable the identification of the printing process parameters in the first place to the operators and implementers of the graphic production process grouped into three process stages of prepress, press and postpress. The graphic production processes are very demanding because of the print runs and the time requirements for printing as a final stage of processing. Therefore, process implementers must respond quickly to all production problems and find solutions to problems in the shortest possible time.

Consequently, a global software system has been developed consisting of subsystems based on algorithmic programming structure, on the principles of expert systems construction and distance learning. The system includes knowledge bases with many parameters. Process deviations also occur as a result of various external influencing parameters such as temperature, humidity, vibration etc. The most commonly used settings during printing are related to the so-called setting position of the printing plates on the cylinders of the carriers of the printing plate. It is common for the amount of ink to change up to $20 \%$ during printing to maintain print quality. The speed of movement of the tabs in the machine is up to $7 \mathrm{~m}$ / s which are very high speeds with which all other process parameters have to be harmonised. It should be handled quickly by obtaining a wealth of information in a short period of time to make the right decision. The graphics systems themselves in the same class have different tuning parameters so that each machine responds differently to the settings in the flow of printing itself. Subsequently, it is necessary to quickly implement appropriate control actions that maintain process stability and print quality.
Through methodological considerations, it was concluded that advanced applications could be linked together to obtain a more complex system with more significant capabilities.

A GRID SIPPS (Serbian language acronym, translated: System of Contemporary Identification of Printing Process Parameters) model was created for these purposes. It provides settings through, algorithms, data models, XML data models, knowledge bases and educational approaches. Microsoft Visual Studio 2010, Databases, Altova XML Spy 2008, Crystal Reports 2008, LPA Prolog and Flex expert system toolkit, Adobe Flash, 3ds Max, Adobe Acrobat 3D and Moodle platforms were used as elements of the development software.

Databases allow organising information logically so the required data can be quickly accessed. They were given the attribute of a knowledge base in the research for the reason that they provide multiple different uses. The Altova XML Spy 2008 is a powerful programming environment, for efficient creation of XML documents. Crystal reports is a report creation environment that is implemented in MS Visual Studio development software. The Prolog software tool has many advantages over other languages and thus, stands out through handling of nonnumeric data, working with datasheets, and a built-in search strategy in the form of automatic returns. Flex is a hybrid expert system's development tool that provides the features and functionality typically associated with expensive expert systems running on artificial intelligence workstations. These features have created opportunities for their implementation in a system improving. Adobe Flash is a multimedia platform primarily introduced by Macromedia, while Adobe Systems does its current development and distribution. Flash is a prevalent method of inserting animations and interactivity into web pages. Its capabilities were used in the methodology of work development. Moodle is a CMS Management Course System (CMS). Moodle (Modular Object-Oriented Dynamic Learning Environment) is one of the most popular and widely-used open-source learning management systems (LMS).

Within the goal set with sub-goals, the examples for specific segments that have the most significant application in the areas of graphical processes that served for the global deployment of the solution model for the overall system were explored and modelled. The particular emphasis of the research was realised through the following stages: development of knowledge bases process with influential offset printing parameters with global concept setting, development of offset printing process model through modelling and construction based on the logic of expert systems, presentation, use and involvement of experts knowledge in decision making with impact assessment of individual parameters by introducing weight coefficients, setting up a complex general model of the printing process parameters identification system of the printing press, development of software system for identification of process parameters of offset printing press using modern software tools based on algorithmic principle with the global model setting, development of software system for identifying process parameters of offset printing press deploying modern distance-based platforms with a worldwide model setup. All development segments are 
complexly laid as the basis of a creative model based on the interactive principle of operation. The full possibilities of constant updating of knowledge bases are opened, especially about process parameters where specific solutions can be obtained, satisfying the required answers. The concept was developed on creative knowledge bases. The idea of the set solution is based on integrative principles, which means that it fits into graphical software designed for various purposes called GRID software. The process parameter identification system is one of the most complex set up systems that connect a large number of knowledge bases. Based on the researched and previously defined substrates, a complex global system model was set up - Fig. 1. The GRID SIPPS global model is conceptually positioned as a segment that uses the level knowledge bases related to the process production phases of graphic production level 1, preparation, printing, finishing graphics processing. Mostly at this level, there are process activities in which problems are identified. The identified issues need to be solved conceptually with a software tool on the second level of the global model with the GRID SIPPS system. Problem identification is essential on this level. In order to solve the issues, it was necessary to explore approaches and approaching opportunities.

Data in databases were obtained experimentally with given environmental conditions that allow repeatability of the research and thus obtained relevant results. The system model is set sufficiently general and could be realised in various software tools. The knowledge base should be supplemented with new research and data related to machines, techniques, process parameters and environmental parameters that are the basis for repeatability of results.

The GRID SIPPS system model consists of three modules that represent conceptual approaches to identifying the process parameters of printing, both individually and as an integrated entity, Fig. 2.

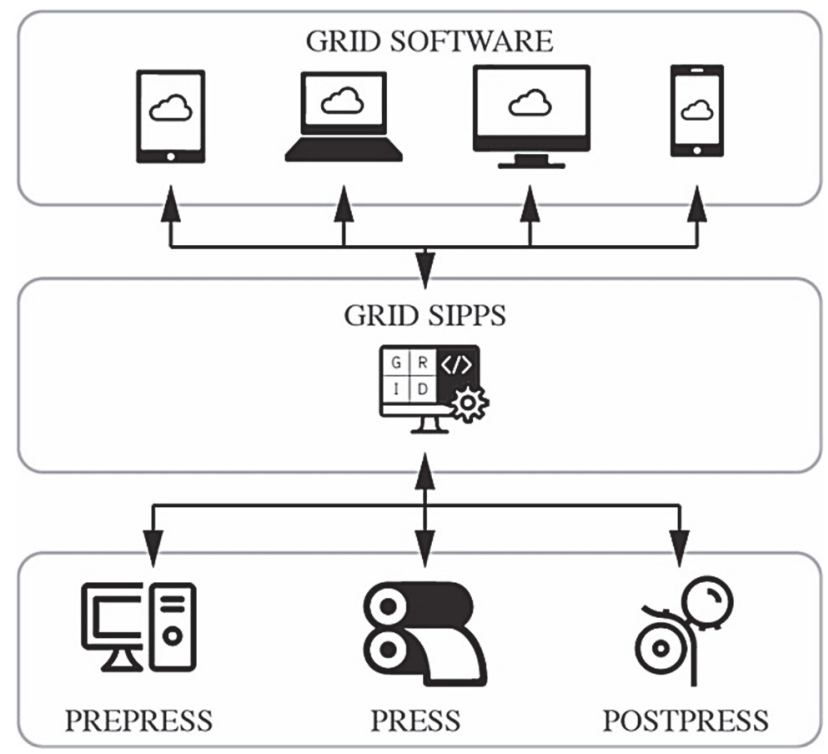

Figure 1 The global model of the GRID system

The GRID SIPPS model system consists of three parts: GRID SIPPS ALG Module, GRID E-Learning Module, GRID ES Module.
The GRID SIPPS ALG module is algorithmically based on enabling data entry on printing techniques, printing machines, printing materials, graphic products, diagnostics, maintenance, connection to the learning module, process parameters and operating system elements.

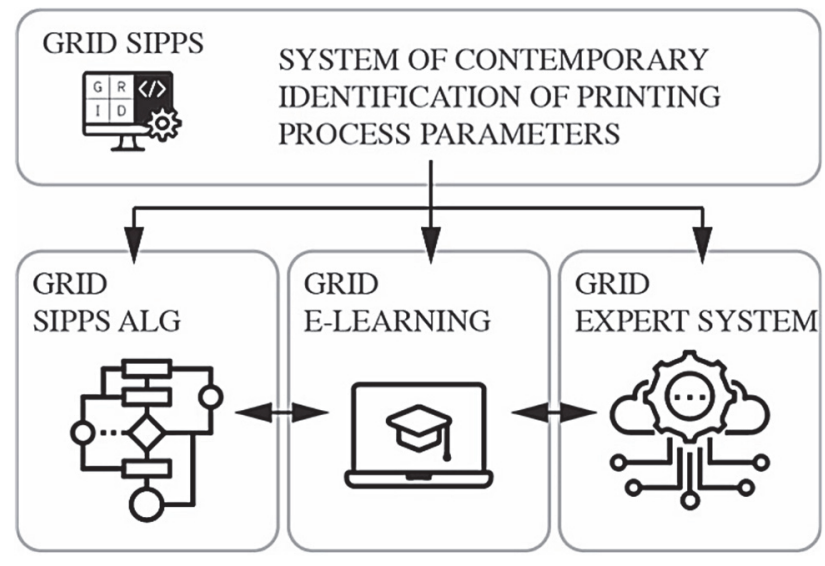

Figure 2 The model of the GRID SIPPS system

GRID E - distance learning module is based on WEBbased systems. It covers the processes of gathering information, researching, creating a knowledge base with structuring knowledge and interacting with users. The education system is user-oriented, where learning is formed according to particular areas of interest for graphic production. Some of the areas they cover are graphic processes, printing techniques, printing forms, packaging, graphic materials, graphic systems, colour management in the reproduction process, workflow automation of production phases, etc. The simulation provides an overview of the operation of the actuators with possible adjustments of the system parameters without the risk of accident or damage that would occur in real conditions. Fig. 3 shows the developed concept of the knowledge base for a graphic machine object with a realistic representation of work visualisation.

The GRID ES module was developed based on the principles of expert systems construction, and it is based on a unique shell of the expert system that can be applied to solving other similar tasks. It consists of the shell and knowledge base of an expert system. While developing GRID ES module, it was sought for universality to:

- develop a universal concept applicable to other modules of the GRID system,

- form a common knowledge base,

- apply the developed solution for solving similar tasks,

- facilitate the development and maintenance of a knowledge base.

In the GRID ES system module, the following has been developed:

- the universal shell of an expert system with comprehensive procedures, rules, representations of knowledge, evaluations, display of outputs for loading and verification of input data from other modules of the GRID system,

- hierarchical knowledge base with structured data, declarative knowledge in the form of facts and procedural - IF-THEN rules, evaluations, input and output data, memory data and their formats. 


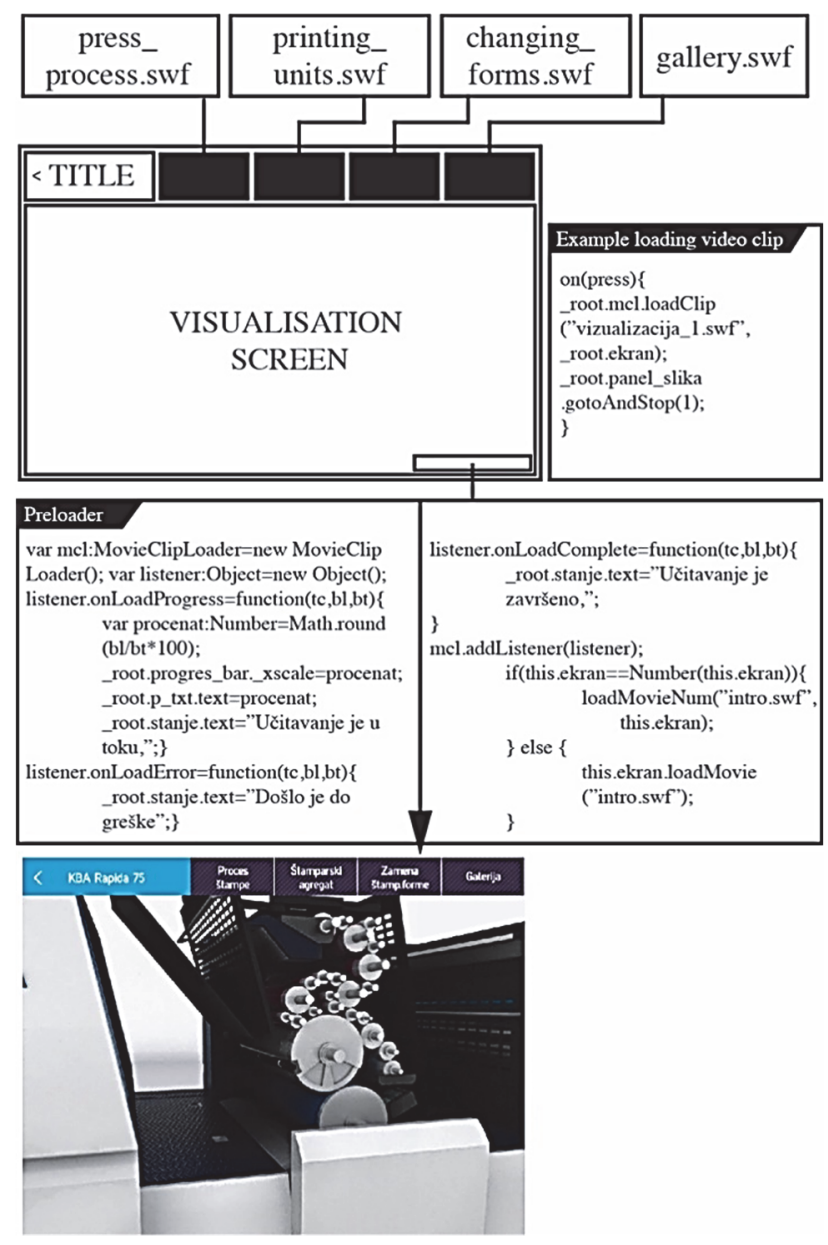

Figure 3 Developed concept of the knowledge base for object

The system for identification of process parameters of printing uses modern approaches of development of expert systems. The developed algorithm is used for manipulating the database and parameters for various segments of graphical processes and systems.

Tables were created to develop knowledge bases. Relations, such as Relationships are used between tables. Crucial primary data is written in tables containing secondary keys, and this data cannot be deleted if it was previously used and stored in the secondary key table. This is the principle of working with keys in Microsoft Access. The links between the tables are given as one-to-many, meaning that data from tables whose primary keys are secondary keys for the other tables, can be used infinitely several times in the other table.

\section{RESULTS AND DISCUSSION}

The research was focused on a software module that provides a significant level of information achieved in the study as well as a large number of results available to the knowledge base. The module settings are aimed at developing or using general-purpose software packages and application software used in the graphics industry. The emphasis in the settings is on the exchange of data between the modules with the application of advanced solutions for testing knowledge and modern approaches to training in modern graphics production systems. E-learning knowledge bases can be linked to educational data. The structure of the e-learning system is intended for a large number of users. The concept with a complete setup requires the division of the system into independent parts between which there is an interactive method of communication. The software part of the system is divided into two segments: data stored on an internet server and user application on a personal computer. The information provided on the Internet server serves to communicate between the database and the application. Communication between the database and the application is via PHP documents that contain commands that enable data exchange. The education system is segmented through modules, where individual modules are created according to separate areas of research of interest for graphic production.

The solution is an information system based on web technologies. Segments of the developed software model for education are an interactive "lab" with a system of graphic machines, which provide different levels of learning from purely transparent description through 2D and $3 \mathrm{D}$ visualisation. Also, an interactive knowledge base (structured through multiple individual modules) with an integrated knowledge test framework for user self-testing and a database of scientific and professional research results, which is continually being updated (Fig. 4).

Multimedia provides the following modalities: multitasking, parallelism and interactivity. Multitasking means that multiple processes are running simultaneously; the parallelism that media can be displayed and executed in parallel; interactivity that hypermedia and hypertexts provide multimedia environments with more natural search capability and user influence on the flow of movement through a digital document.

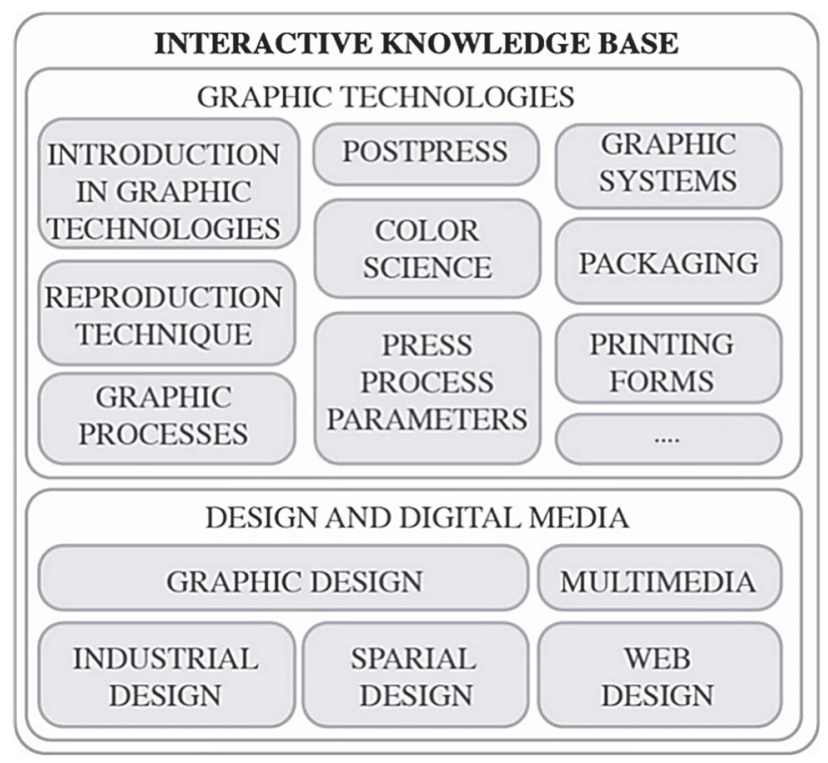

Figure 4 The organisational structure of the knowledge base

Based on web technologies, the education system also allows searching and downloading materials from the knowledge base and the database of scientific and professional papers, as well as checking the acquired knowledge by solving tests from mobile devices (smartphones, tablets).

Within the development of knowledge bases for visualisation of system functioning, the concept of the online interactive laboratory was developed in which 
visualisations of individual graphics systems were integrated, showing the principle of operation, system components, subsystems and circuits.

Simulations of the work of graphical systems have been developed which provide experience-based refinement with a better understanding of the process and the development of solving skills. Unlike operators who solve production problems based on years of experience, machine simulators reduce time and training costs by varying settings such as machine parameter values, error analysis, potential issues, and suggestions for optimal solutions.

Visualisation is the process of creating research objects from different environments into a presentation device. It is a way to make visible everything impossible to see in a graphical system in a real environment. The visualisation that enables realistic display, manipulation and decomposition of complex graphics systems was realised using Adobe Flash software with Action Script programming language for $2 \mathrm{D}$ modelling with interactive elements in Autodesk 3Ds Max Studio for 3D modelling.

Fig. 5 shows the developed visualisation process with the simulation of the graphics system operation.

Microsoft Visual Basic 2010 Ultimate, Microsoft Access 2010, Altova XML Spy 2008, AltovaStyleVision 2008, Crystal Reports 9, Adobe Acrobat DC, LPA Prolog and Flex Expert System Toolkit, 3ds Max Design 2014, Adobe Flash CS6, Moodle Platform, Simlab Composer 2015, Adobe Illustrator CS6 and Adobe Photoshop CS6 are applied software development tools.

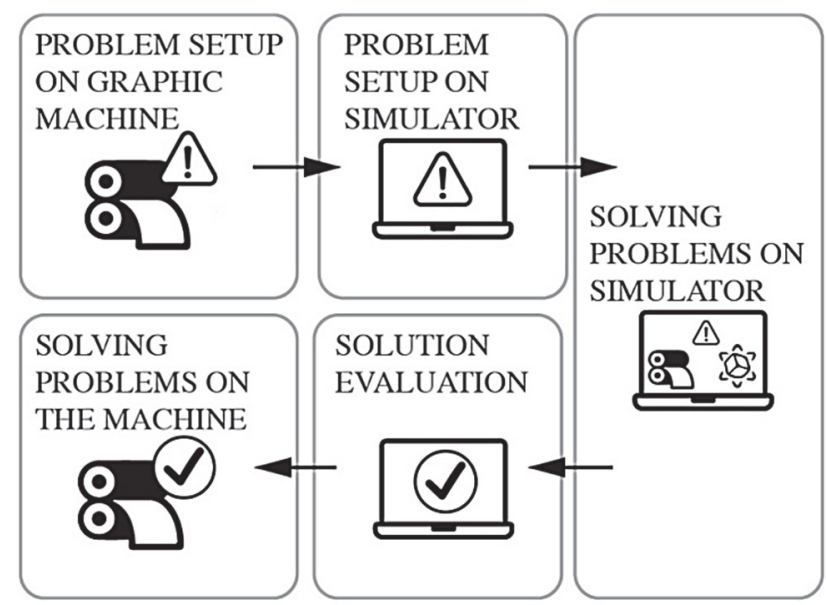

Figure 5 Visualisation process developed with the simulation of graphic system operation

The GRID SIPPS ALG module is a part of the overall GRID SIPPS system, (Fig. 6) designed in the way that it can be a functional entity integrated into the global model as a module.

Based on a detailed analysis of all the influential parameters, a model was made for developing a modern solution for identifying the offset printing process parameters of the GRID, which is shown in Fig. 7. Accordingly, specific tasks were set up that produce the desired results. These solutions are based on setting up a complex general model of the system for identification of process parameters of printing, identification of the most influential process parameters of tabbed offset printing, creation of models of the process of offset printing through modelling of the most influential parameters, development of a software system for identification of process parameters of offset printing press using modern software tools-modular conception.

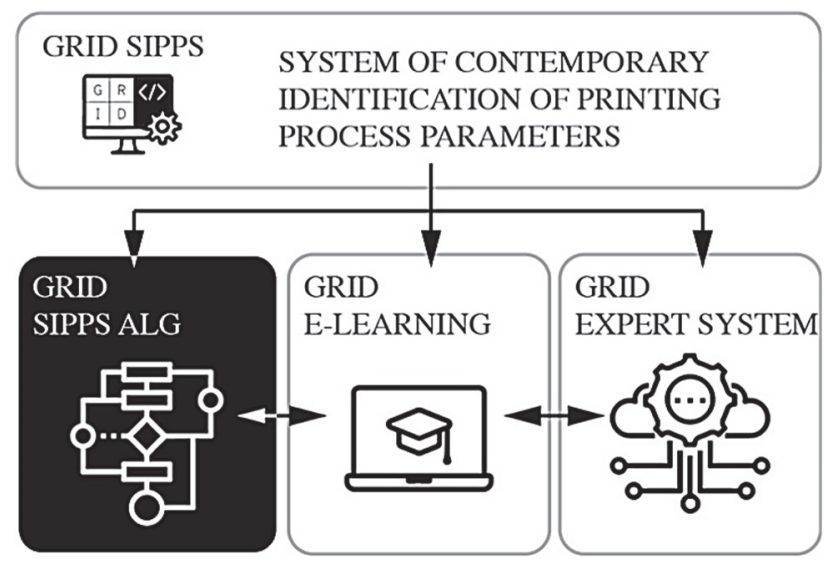

Figure 6 The module GRID SIPPS ALG

The concept of a modern software system is based on the most significant parameters that have the greatest impact on print quality. It was developed to have the ability to enter new parameters by the user so that the parameter range can be expanded. The system was developed, so the user has the possibility of education based on knowledge of the influence of specific parameters on the print quality.

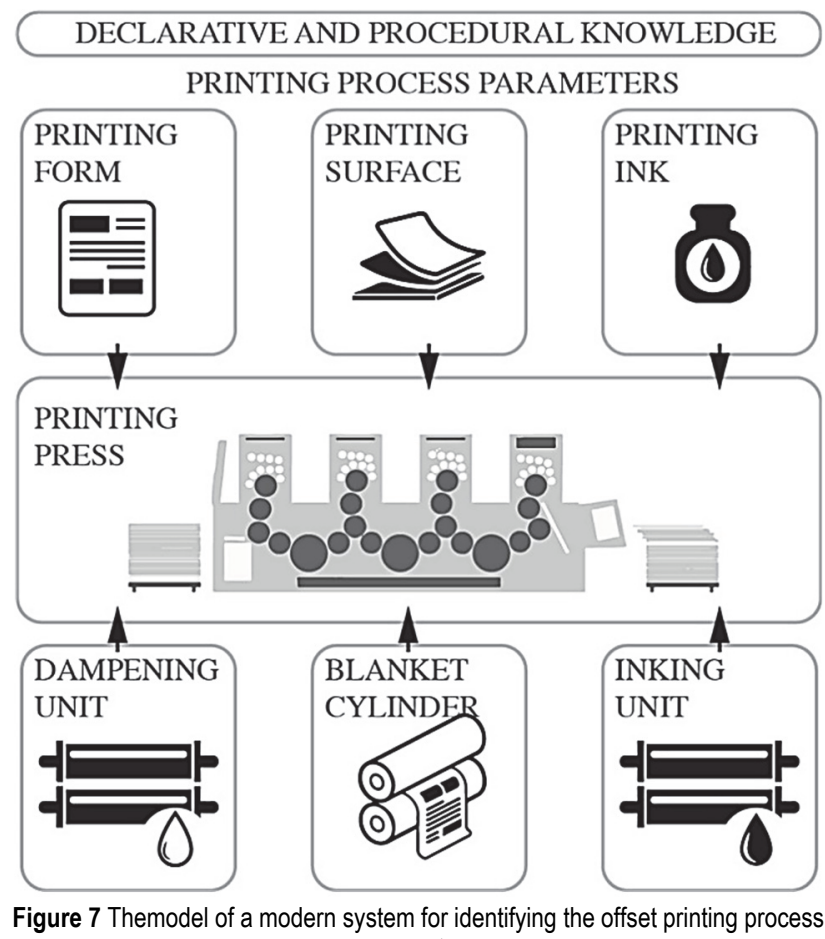
parameters

As the knowledge base is expandable, some new insights can be introduced to give the user more significant educational opportunities. Adding new insights into the impact of individual parameters on print quality-related to user education is done by modifying an existing XML document. The user must have an internet browser installed to view these documents, while for editing the documents it is necessary to have some XML editor. 
The database of the GRID SIPPS ALG system should ensure the following: data can be entered, changed or deleted, searching for the desired data, learning based on knowledge of the impact of particular parameters, the knowledge base is extensible, i.e. to bring in some new insights from users.

Accordingly, a relational database was set up to meet the stated requirements of Fig. 8.

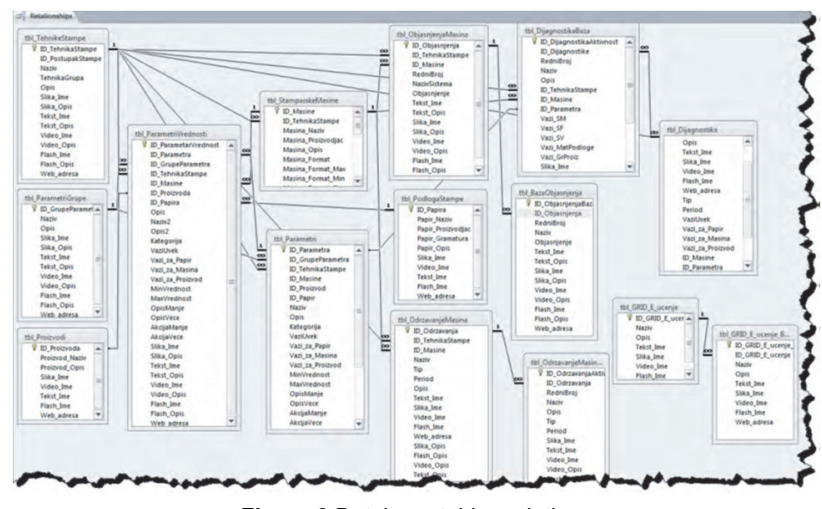

Figure 8 Database tables relations

Microsoft Access 2010, which is a part of the extensive Microsoft Office suite, was selected as the DBMS system. One of the reasons for choosing MS Access 2010 is that the standards that apply to MS Windows and other MS Office programs also apply to MS Access, such as a fully standardised user interface, intuitive usage, unified procedures and functions, etc.

\section{CONCLUSION}

The information system model that has been developed integrates new technologies into a single unit with aims to improve communication and information distribution between parts of a graphics production system. Unlike the existing models that operate on conventional communication systems, this information system model integrates modern communication technologies to facilitate communication and better-informing individuals involved in the process of graphic production. The significance of this solution would be reflected in the developed research methodology of importance for scientific conclusions. This solution can be applied in the education and machine operators education processes, as well as in the graphic production processes.

The analysis of the given literature sources presented in the paper concluded that each of the analyzed systems has specific approaches and logic in development. The developed solution given in the paper is original and comprehensive. The presented results are derived from the original approach to the development of a software solution based on the principles of artificial intelligence based on modern software applications intended for the development of expert systems. The developed system incorporates the modern logic of algorithmic structures as well as the logic of e-learning, which significantly increases the reliability of the developed knowledge base.

\section{Acknowledgements}

This research (paper) has been supported by the Ministry of Education, Science and Technological Development through the project No. 451-03-68/202014/200156: "Innovative scientific and artistic research from the FTS (activity) domain".

\section{REFERENCES}

[1] Kašiković, N., Novaković D., Milić N., Vladić G., Zeljković, Ž., \& Stančić, M. (2015). Thermovision and Spectrophotometric Analysis of Ink Volume and Material Characteristics Influence on Colour Changes of Heat Treated Printed Substrates. Tehnicki vjesnik - Technical Gazette, 22(1), 33-41. https://doi.org/10.17559/TV-20130928115500

[2] Pal, M., Novaković, D., Dedijer, S., Koltai, L., Jurič, I., Vladić, G., \& Kašiković, N. (2017). Image processing based quality control of coated paper folding. Measurement, 100, 99-109. https://doi.org/10.1016/j.measurement.2016.12.033

[3] Novaković, D. \& Avramović, D. (2012). Influence of printing surface attributes on print quality in electrophotography. Tehnicki vjesnik - Technical Gazette, 19(2), 295-301

[4] Pavlović, Ž., Dedijer S., Kašiković N., Novaković, D., Pal M., \& Risović, D. (2017). Evaluation of effective models of offset CTP plate aluminium oxide surface roughness characterization. International Journal of Surface Science and Engineering, 11(5), 433-449. https://doi.org/10.1504/IJSURFSE.2017.088129

[5] Dedijer, S., Pal, M., Boeva, R., Spiridonov, I., Bozhkova, T., Zorić, V., \& Zeljković, Ž. (2017). Characterization of flexo and letterpress printing plate's surface roughness by indirect SEM image-based profilometry. Bulgarian Chemical Communications, 49, 148-157.

[6] Delić, G., Vladić, G., Pál, M., Banjanin, B., \& Dedijer, S. (2017). Performance evaluation of paper embossing tools produced by fused deposition modelling additive manufacturing technology. Journal of graphic engineering and design, 8(2), 47-54. https://doi.org/10.24867/JGED-2017-2-047

[7] Dinesh, Y., Deepak., C., Ramesh Kumar, G., Akash, A., \& Ashish, P. (2020) Optimization of FDM 3D printing process parameters for multi-material using artificial neural network. Materials Today: Proceedings, 21, 1583-1591. https://doi.org/10.1016/j.matpr.2019.11.225

[8] Kiphan, H. (2001). Handbook of Print Media: Technologies and Production Methods. Springer-Verlag, Berlin Heidelberg New York.

[9] Almutawa, S. \& Moon, Y. B. (1999). The development of a connectionist expert system for compensation of color deviation in offset lithographic printing. Artificial Intelligence in Engineering, 13, 427-434. https://doi.org/10.1016/S0954-1810(99)00019-9

[10] Bergman, L. (2005). Using multicoloured halftone screens for offset print quality monitoring. $\mathrm{PhD}$, Intelligent Systems Laboratory, School of Information Science, Computer and Electrical Engineering, Halmstad University, Halmstad, Sweden.

[11] Tchan, J., Thompson, R. C., \& Manning, A. (1999). A computational model of print-quality perception. Expert Systems with Applications, 17(4), 243-256. https://doi.org/10.1016/S0957-4174(99)00038-X

[12] Englund, C. \& Verikas, A. (2008). Ink flow control by multiple models in an offset lithographic printing process. Computers \& Industrial Engineering. https://doi.org/10.1016/j.cie.2008.01.019 
[13] Lundström, J. (2014). Situation Awareness in Colour Printing and Beyond. PhD, Halmstad University Dissertations No. 6, Halmstad University Press.

[14] Shahin, A. (2016). State-of-the-art review of some artificial intelligence applications in pile foundations. Geoscience Frontiers, 7, 33-44. https://doi.org/10.1016/j.gsf.2014.10.002

[15] Liao, S. H. (2005). Expert system methodologies and applications - a decade review from 1995 to 2004. Expert Systems with Applications, 28, 93-103. https://doi.org/10.1016/j.eswa.2004.08.003

[16] Bergman, L. \& Verikas, A. (2004). Intelligent Monitoring of the Offset Printing Process. Neural Networks and Computational Intelligence proceeding, 173-178.

[17] Acosta, G. \& Todorovich, E. (2003). Genetic algorithms and fuzzy control: a practical synergism for industrial applications. Computers in Industry, 52, 83-195. https://doi.org/10.1016/S0166-3615(03)00102-7

[18] Li, B., Hou, B., Yu, W., Lu, X., \& Yang, C. (2017). Applications of artificial intelligence in intelligent manufacturing: A review. Front Inform. Tech. El., 18(1), 8696. https://doi.org/10.1631/FITEE.1601885

[19] Varepo, L., Trapeznikova, O., Panichkin, A., Roev, B., \& Kulikov, G. (2018). Software for Quantitative Estimation of Coefficients of Ink Transfer on the Printed Substrate in Offset Printing. Journal of Physics: Conference Series, 998. https://doi.org/10.1088/1742-6596/998/1/012041

[20] Orlova, E., Varepo, L. G., \& Hodes, A. (2020). Diagnostics of paper - dampening solution printing system parameters for open source software applications. Journal of Physics: Conference Series, 1546. https://doi.org/1088/1742-6596/1546/1/012023

[21] Villalba-Diez, J., Schmidt, D., Gevers, R., Ordieres-Meré, J., Buchwitz, M., \& Wellbrock, W. (2019). Deep Learning for Industrial Computer Vision Quality Control in the Printing Industry 4.0. Sensors, 19, 3987. https://doi.org/10.3390/s19183987

[22] Zhang, E., Chen, Y.,Gao, M., Duan, J., \& Jing, C. (2019). Automatic defect detection for web offset printing based on machine vision. Applied Science, 9, 3598. https://doi.org/10.3390/app9173598

[23] He, B. \& Bai, K. J. (2020). Digital twin-based sustainable intelligent manufacturing: a review. The International Journal of Advanced Manufacturing Technology. https://doi.org/10.1007/s40436-020-00302-5

[24] Chen, Y., He, P., Gao, M., \& Zhang, E. (2019). Automatic Feature Region Searching Algorithm for Image Registration in Printing Defect Inspection Systems. Applied Science, 9, 4838. https://doi.org/10.3390/app9224838

[25] Zhang, E., Li, B, Li, P., \& Chen, Y. (2019). A Deep Learning Based Printing Defect Classification Method with Imbalanced Samples. Symmetry, 11, 1440. https://doi.org/10.3390/sym11121440

[26] Alom, M. Z., Taha, T. M., Yakopcic, C., Westberg, S., Sidike, P., Nasrin, M. S., Hasan, M., Van Essen, B. C., Awwal, A. A. S., \& Asari, V. K. (2019). A State-of-the-Art Survey on Deep Learning Theory and Architectures. Electronics, 8, 292. https://doi.org/10.3390/electronics 8030292

\section{Contact information:}

Željko ZELJKOVIĆ, PhD

(Corresponding author)

University of Novi Sad, Faculty of Technical Sciences, Department of Graphic Engineering and Design,

Trg Dositeja Obradovića 6, 21000 Novi Sad, Serbia

E-mail: zeljkoz@uns.ac.rs

Nemanja KAŠIKOVIĆ, PhD

University of Novi Sad, Faculty of Technical Sciences,

Department of Graphic Engineering and Design,

Trg Dositeja Obradovića 6, 21000 Novi Sad, Serbia

E-mail:knemanja@uns.ac.rs

Stefan Đurđević, PhD

University of Novi Sad, Faculty of Technical Sciences, Department of Graphic Engineering and Design,

Trg Dositeja Obradovića 6, 21000 Novi Sad, Serbia

E-mail: djurdjevic@uns.ac.rs

Dragoljub Novaković, PhD

University of Novi Sad, Faculty of Technical Sciences, Department of Graphic Engineering and Design,

Trg Dositeja Obradovića 6, 21000 Novi Sad, Serbia

E-mail: novakd@uns.ac.rs 